Article

\title{
Wind Tunnel Studies of a Pedestrian-Level Wind Environment in a Street Canyon between a High-Rise Building with a Podium and Low-Level Attached Houses
}

\section{Chien-Yuan Kuo ${ }^{1,2}$, Chun-Ta Tzeng ${ }^{1}$, Ming-Chin Ho ${ }^{2}$ and Chi-Ming Lai ${ }^{3, *}$}

1 Department of Architecture, National Cheng Kung University, No. 1, University Road, Tainan 701, Taiwan; E-Mails: kcy@abri.gov.tw (C.-Y.K.); ctmt@mail.ncku.edu.tw (C.-T.T.)

2 Architecture and Building Research Institute, Ministry of the Interior, No. 200, Sec. 3, Beisin Rd., Sindian District, New Taipei 23143, Taiwan; E-Mail: ho@abri.gov.tw

3 Department of Civil Engineering, National Cheng Kung University, No. 1, University Road, Tainan 701, Taiwan

* Author to whom correspondence should be addressed; E-Mail: cmlai@mail.ncku.edu.tw; Tel.: +886-6275-7575 (ext. 63136); Fax: +886-6209-0569.

Academic Editor: Nyuk Hien Wong

Received: 3 July 2015 / Accepted: 21 September 2015 / Published: 29 September 2015

\begin{abstract}
The pedestrian-level wind environment quality in street canyons formed by high-rise buildings and other low-level buildings could be affected by multiple factors, such as the height and geometry of the surrounding buildings, street width, wind direction, and wind speed. This study conducted wind tunnel experiments to determine the characteristics of the pedestrian-level wind environment in street canyons under different conditions, including different street widths, podium heights, and approaching wind directions. The experimental results revealed that the effects of the street canyon width on the pedestrian-level wind in a street canyon can be categorized into three different flow regimes. A higher podium creates stronger wind speeds of the flow within the street canyon, and different approaching wind directions change the high-wind-speed zone within the street canyon accordingly.
\end{abstract}

Keywords: pedestrian-level wind; street canyon; high-rise building; podium; wind tunnel 


\section{Introduction}

Limited city land resources approach saturation with economic development. Building contractors attempt to satisfy the limits of building codes, while using land efficiently. Building functions with high usage intensity will typically be at the lower floor with a flat and wide spatial volume to reduce the use of vertical moving lines, whereas other building functions would be placed at higher floors. Thus, an uplifting basis (podium) is formed, further extending the height of a high-rise tower. This is a common building pattern for residential or commercial uses in metropolitan areas. The buildings next to high-rise buildings are largely low-level attached houses. The street canyon formed between both sides of the buildings could be a boulevard $30 \mathrm{~m}$ in width or could be an $8-\mathrm{m}$ alley. Different street canyon widths will form different wind environment characteristics. The architectural design must consider the safety and aesthetics as well as the effect of the building on the microclimate. The pedestrian-level strong winds produced by high-rise buildings could pose problems for pedestrian safety and comfort. This issue has gained widespread attention, and there are many studies that explore related problems.

The process of an outdoor wind flow being blocked or guided by high-rise buildings and then moved toward a street canyon until it flows out at a street corner forms complex air flow patterns that are interactively affected by many factors. Due to the channeling effect inside the street canyon, the wind speed inside the street canyon will be accelerated and then cause an instantaneous strong wind that is a nuisance. The wind flow characteristics inside the street canyon are affected by the approaching wind direction, the width of the street canyon, and the heights of the buildings on both sides.

To and Lam [1] configured tested cases into three types: A single building, forward or backward arrangements along the wind direction, and right or left arrangements across the wind direction. Then, they adopted a street canyon with a fixed width to conduct wind tunnel tests to understand the pedestrian-level wind characteristics in a street canyon. Their study indicated that the forward and backward arrangement had smaller effects on the pedestrian-level wind. However, the wind speed in the street canyon was notably accelerated when taking the left or right arrangement.

Stathopoulos et al. [2] made four types of arrangements for wind tunnel testing: An isolated building, an isolated building with an open bottom, forward and back arrangement of two buildings, and right and left arrangement of two buildings. The configuration model that is relevant to the present study is the right and left arrangement of two buildings; their study took four different building dimensions to allow wind tunnel testing of eight different street canyon widths to explore the pedestrian-level wind characteristics. The results showed that the maximum wind speed occurred when $L / S=0.4$ ( $L$ is the street width; $S$ is a building section factor).

In addition to laboratory-scale experiments, computational fluid dynamics (CFD) simulations have also been widely used to investigate street canyon airflow. Blocken et al. [3] conducted studies with CFD simulations and the aforementioned literature [2] on wind tunnel testing and proposed three types of passage flow patterns: resistance flow, interaction flow, and isolation flow. The study indicated that the street wind speed increased only at the pedestrian level; the wind speed of other heights only increased by $8 \%$ compared to the free flow condition (no building); therefore, it is not a good indication that the street wind flow meets the Venturi effect. 
Blocken et al. [4] configured two long and narrow buildings into diverging and converging types; in the wind tunnel testing, they simulated the open terrain wind speed profile to understand the wind passage. The results showed that the wind speed of the diverging passage was higher than that of the converging passage and that the wind speed increased with increasing street canyon width. This finding contradicts the past study results of parallel street canyons. In addition, the wind speed of the diverging passage is at a maximum when the wind direction is $0^{\circ}$. The maximum wind speed of the converging passage occurs when the wind direction is $15^{\circ}$.

Tsang et al. [5] explored the effects of different building dimensions, spacing arrangements, and podiums on the pedestrian-level wind environment around buildings. The arrangement spacing was set at four parallel buildings with dimensions of $25 \mathrm{~m}$ (width) $\times 25 \mathrm{~m}$ (depth) $\times 125 \mathrm{~m}$ (height), and the adjacent spacing of $0,6.25,12.5,18.75$, and $25 \mathrm{~m}$ for the wind tunnel testing. Their results showed that the maximum dimensionless wind speeds in the street canyon were 1.06, 1.0, 1.12, 1.15, and 1.11 for the spacings of $0,6.25,12.5,18.75$, and $25 \mathrm{~m}$, respectively. The high-wind-speed zones were all present on the side of the isolated buildings or inside the passage of two adjacent buildings. The study also found that a wider street canyon can effectively improve the pedestrian-level strong wind in the street canyon.

$\mathrm{Gu}$ et al. [6] configured uneven building layouts and non-uniform street canyons and used CFD simulations to study the effects of building layouts on the wind environment in non-uniform street canyons under various building arrangements. The results show that the air flows in the non-uniform street canyons are more complex than in the uniform street canyon. The titling, horizontal divergence and convergence of wind streamlines are observed inside the non-uniform street canyons.

Hang et al. [7] arranged nine-row and 18-row high-rise square arrays and simulated idealized high-rise urban areas. They sought to understand how the building heights affect the pollutant dispersion and pedestrian ventilation. The CFD simulation results showed that the pollutant removal mainly depends on the mean flows for nine-row arrays with building height variations (standard deviations of $0 \%-57.1 \%$ ) and the average canopy height. Larger standard deviations tend to induce better pedestrian ventilation.

Moon et al. [8] used CFD simulations to investigate the flow characteristics of the complex urban street canyon around high-rise buildings. Their results showed that wind directions dominate the flow characteristics in the street canyons. When winds blow obliquely or perpendicular to the street canyon, jet-like flows are found between tall buildings along the street canyon. When winds blow parallel to the street canyon, the mean velocity in the street canyon is maintained at a moderate level.

Ramponi et al. [9] investigated the outdoor ventilation of generic urban configurations with different wind directions on equal and unequal street widths via CFD. Their results showed that the main street can be both beneficial and non-beneficial for the ventilation efficiency of the downstream area depending on the wind directions. For wind oblique or perpendicular to the main street, the presence of the main street generally increases the ventilation efficiency because it acts as a sink for clean air. However, the parallel wind direction, where the higher flow rate through the main street reduces the flow rate through the parallel narrower streets, negatively affects the ventilation efficiency.

This study conducted wind tunnel experiments to explore the pedestrian-level wind characteristics in the street canyon formed by a high-rise building with a podium and the adjacent low-rise attached houses under different podium heights, street canyon widths, and approaching wind directions. 
Regarding street canyon ventilation research, many studies in the literature took building patterns with symmetrical sections on two sides as the research object and only explored the wind speed of the center position of street canyons. This study considered the wind flow guided by asymmetrical building sections that are formed by high-rise buildings containing a podium and their adjacent attached blocks. Moreover, this study conducted wind speed measurements in many places, including the front of the street canyon, inside the street canyon, and downstream of the street canyon, to fully understand the flow around the two adjacent buildings.

\section{Research Method}

\subsection{Experimental Setup}

The experiments were conducted at the Wind Tunnel Laboratory, Architecture and Building Research Institute, Ministry of the Interior, Taiwan, located on the Gueiren Campus of National Cheng-Kung University. As shown in Figure 1a, the wind tunnel is a closed type that has two test sections. The first test section is equipped with two rotating discs.

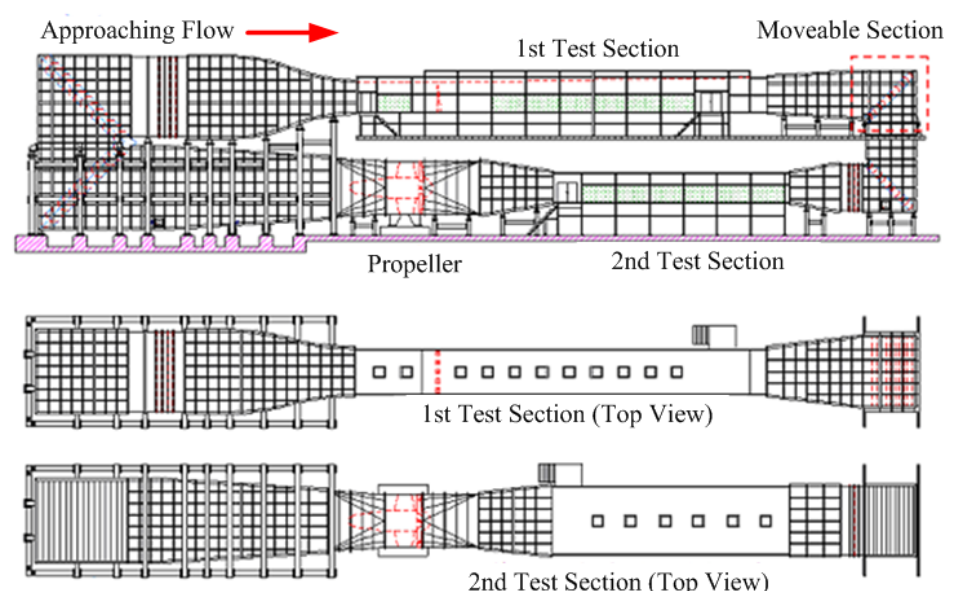

(a)

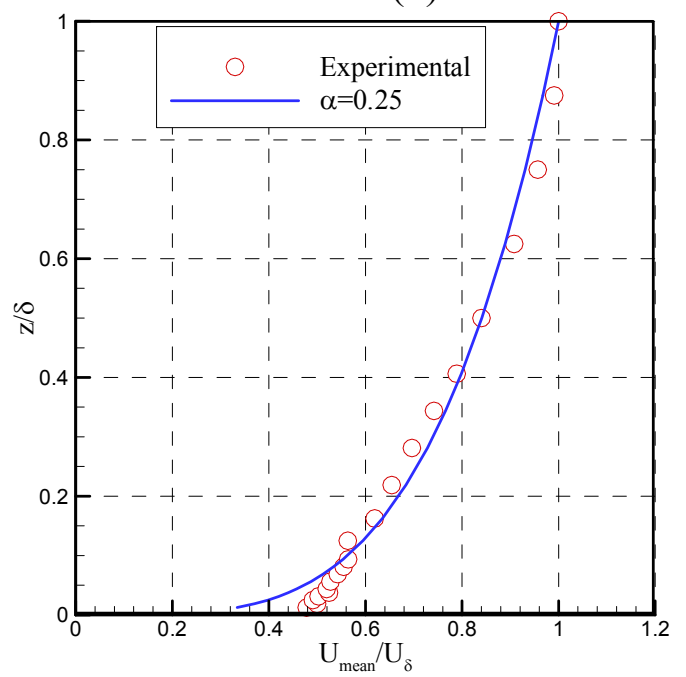

(c)

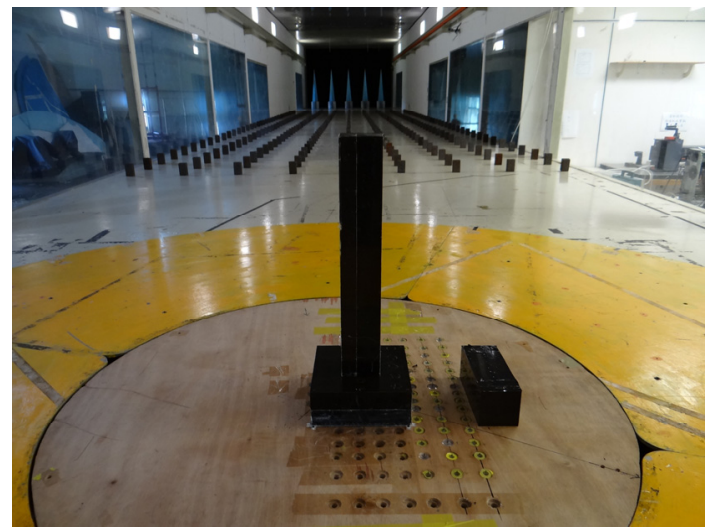

(b)

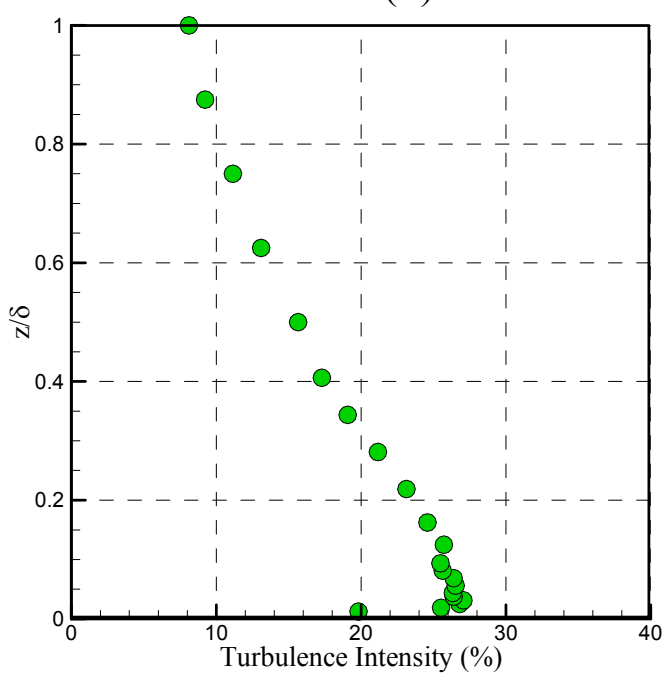

(d)

Figure 1. (a) Schematic diagrams and (b) photo of the wind tunnel; (c) mean speed profile and (d) turbulence intensity approaching wind profile in the wind tunnel. 
The diameter of the first rotating disc is $1 \mathrm{~m}$ and is placed at a distance of $3 \mathrm{~m}$ from the entrance of the test section to undertake a measurement of general fluid dynamics. The diameter of the second rotating disc is $2.6 \mathrm{~m}$ and is placed on a movable track positioned at a distance between $25.5 \mathrm{~m}$ and $31.5 \mathrm{~m}$ from the entrance edge. This disc is mechanically controlled to perform rotation as well as up and down motions and is mainly used to conduct aerodynamics studies of wind action on buildings and pollution diffusion testing. The second test section is equipped with a rotating disc located $15 \mathrm{~m}$ from the exit of the rectification section in the wind tunnel. The turntable has a diameter of $2.6 \mathrm{~m}$ and is mainly used for bridge testing. The pedestrian-level wind flow testing of this study was conducted using the second rotating disc of the wind tunnel's first test section. With a maximum wind speed of $30 \mathrm{~m} / \mathrm{s}$, this test section had a length of $36.5 \mathrm{~m}$, a width of $4 \mathrm{~m}$, and a height of $2.6 \mathrm{~m}$.

According to the related regulation in Taiwan [10], the mean wind speed profile of the approaching turbulent wind flow in the wind tunnel follows a power law exponent of 0.25 , using a series of $1.6 \mathrm{~m}$ spoiler and roughness elements, to simulate wind flow above suburban terrain. The building models were fabricated at a length scale of $1 / 250$. Figure $1 \mathrm{c}, \mathrm{d}$ shows the normalized mean wind speed, $U_{\text {mean }} / U_{\delta}$, and turbulence intensity of the approaching wind in the wind tunnel, respectively. $U_{\text {mean }}$ is the mean wind speed at the height of $z ; U_{\delta}$ is the mean wind speed of the modeled atmospheric boundary layer height $(\delta=1.6 \mathrm{~m})$.

\subsection{Experimental Model}

Because pedestrian-level wind speeds in and around the street canyon are the main investigated objective, a rigid experimental model was adopted. The total height of the experimental model was fixed $\left(H+P_{\mathrm{h}}=56 \mathrm{~cm}\right) . H$ and $P_{\mathrm{h}}$ are the tower height and podium height, respectively. The length and width of the podium and the length, width, and height of the adjacent attached houses were fixed. The variables include the podium height, street canyon width, and approaching wind direction. Layouts of the experimental model and surface wind sensors are shown in Figure 2. The model dimension, $D$, was $8 \mathrm{~cm}$; the podium width was set to $2.5 D$, and the podium height ratio, $P \mathrm{~h} / D$, was set to $0.5,1$, and $2 . S$ is the width of street canyon, and the street canyon spacing ratios, $S / D$, were set to $0.375,0.75,1.25,1.75$, and 2.25 . The approaching wind angles were $\theta=45^{\circ}, 22.5^{\circ}, 0^{\circ},-22.5^{\circ}$, and $-45^{\circ}$.

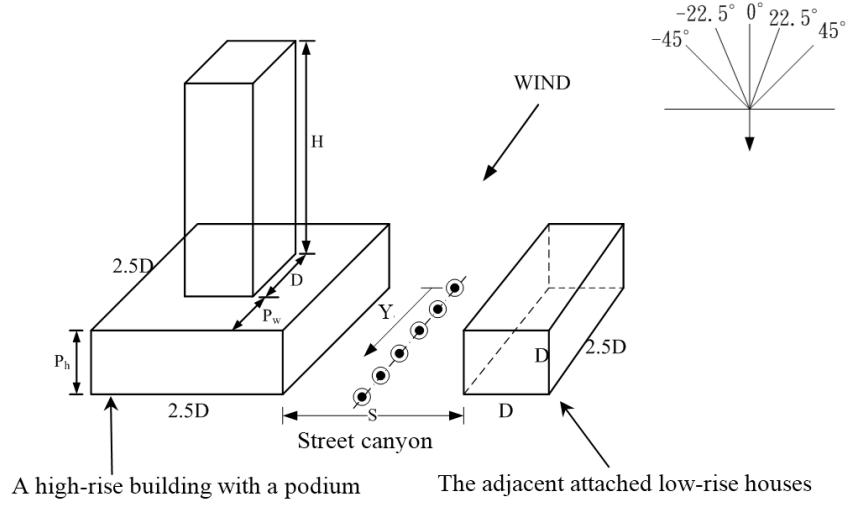

(a)

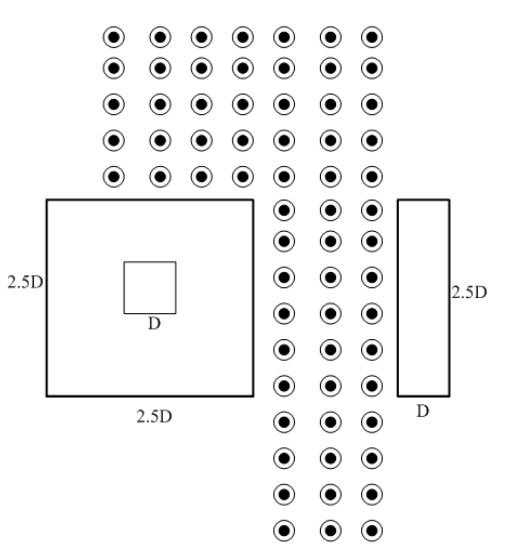

(b)

Figure 2. Experimental model (a) and surface wind sensor deployment (b). 
The layout modes of the surface wind sensors (Irwin probe) included the front area of the podium model, upstream of the street canyon, inside the street canyon, and downstream of the street canyon; a total of 60 measurement points were placed. The outer diameter of the Irwin probe was $2 \mathrm{~cm}$, so it was not possible to lay out these points for more than two rows when $S=0.375 D$ and $S=0.75 D$. Hence, single-row layouts were created only on the center row for these two street canyon widths. As shown in Figure 2, $S=1.25 D, S=1.75 D$, and $S=2.25 D$ were all laid out with 3 rows, which are each spaced by $5 \mathrm{~cm}$ to the left and right. For the 6 measurement points laid along the wind direction inside the street canyon, the positions were set at $Y / D=0,0.5,1,1.5,2$, and 2.5.

\subsection{Surface Wind Sensor (Irwin Probe)}

A surface wind sensor (Irwin probe) is a simple and omnidirectional piece of equipment that can be used to measure both the mean wind speed and lower-frequency fluctuations of pedestrian-level winds in wind tunnel testing. Because of its axisymmetry, it requires no alignment with the flow direction [11]. Compared to other anemometers, an Irwin probe has a small volume and can be easily fixed onto the surface; therefore, multi-point and wide range wind speed measurements can be conducted while not affecting the main wind flow patterns. The Irwin probe has been proven to be satisfactory for practical use in wind tunnel studies [5,12-14].

Each Irwin probe consists of a sensor tube that protrudes above the modeled ground level to a desired height that is mounted in a round sensor hole. The pressure difference between the sensor hole and the top of the sensor tube was measured to calculate the wind speed at that desired height. A simplified relation between the pressure difference, $\Delta P$, and the wind speed, $u$, was given by Irwin [11]:

$$
u=\alpha+\beta \sqrt{\Delta P}
$$

The calibration constants $\alpha$ and $\beta$ in Equation (1) were determined using the Cobra Probes (Turbulent Flow Instrumentation Pty Ltd., Victoria, Australia). Then, a hotwire anemometer and Irwin probe were installed together inside the wind tunnel at a common height above the floor of the wind tunnel. Good agreement can be observed between the Irwin probe measurements and hotwire anemometer measurements under 16 different wind velocities (data not shown).

\section{Results and Discussion}

All of the study outcomes use the free stream wind speed outside the modeled atmospheric boundary layer as the reference speed $\left(U_{\mathrm{h}}\right)$; the mean wind speed of each measurement point was divided by $U_{\mathrm{h}}$ to obtain the dimensionless mean wind speed of each measurement point $\left(U_{\mathrm{i}} / U_{\mathrm{h}}\right)$.

\subsection{Effect of the Street Canyon Width}

The pedestrian-level wind flows of the street canyon center position are shown in Figure 3. Dimensionless mean wind speed and root-mean-square (RMS) results of the case with $S=0.375 D$ are provided in Table 1. 


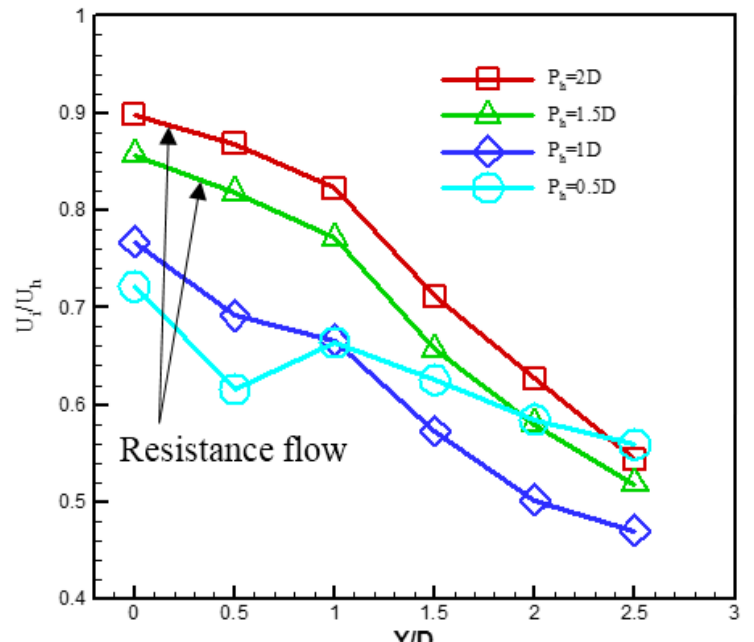

(a)

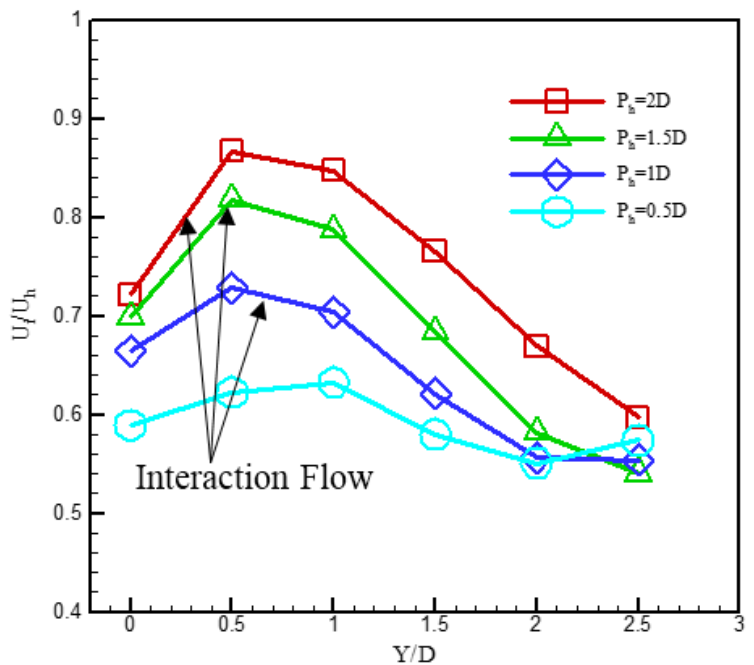

(c)

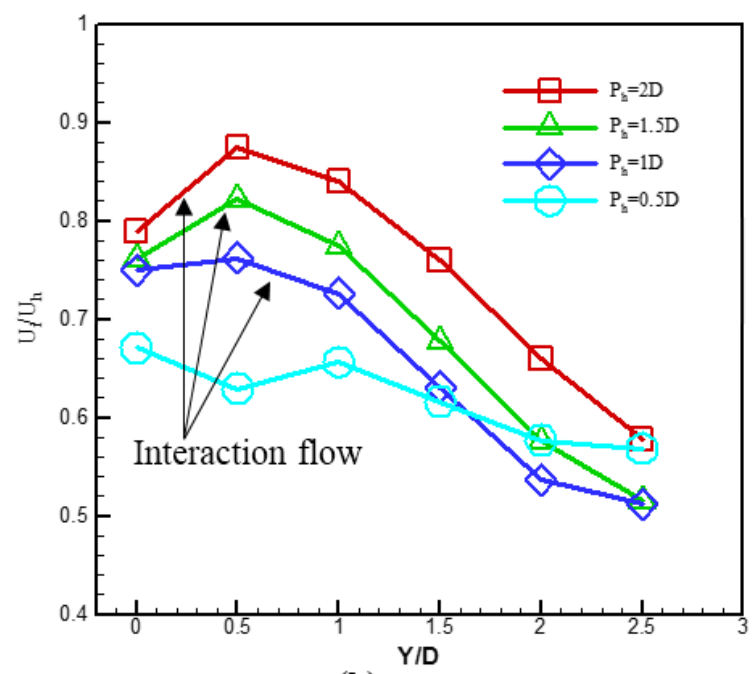

(b)

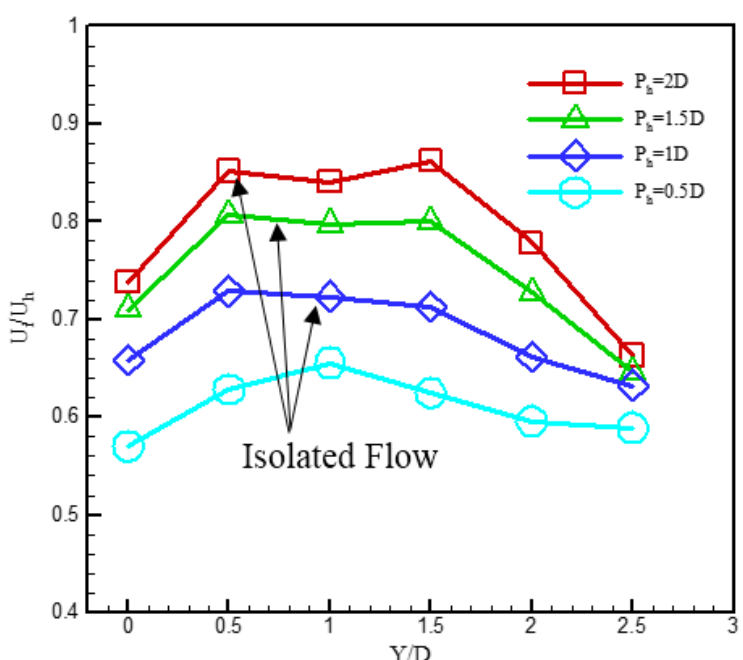

(d)

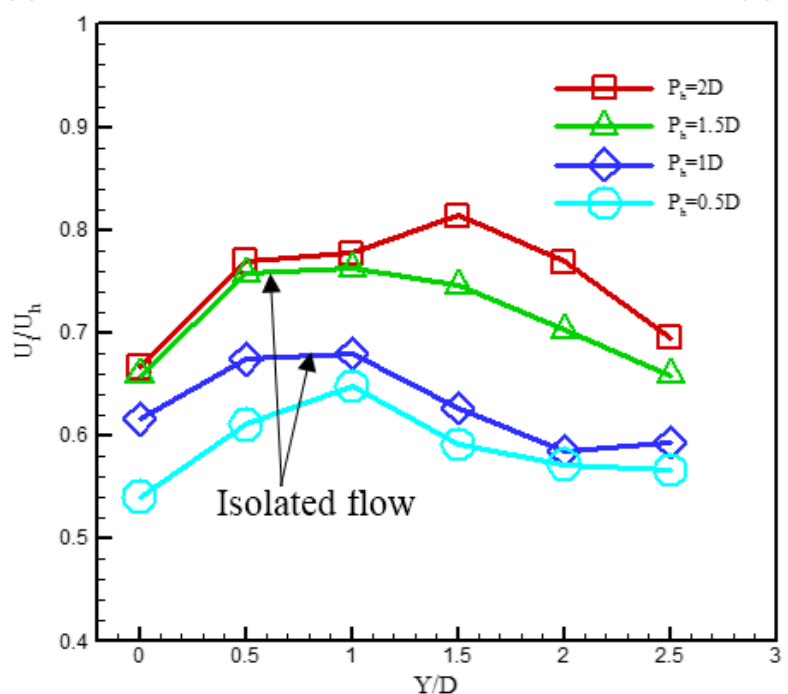

(e)

Figure 3. (a) $S=0.375 D$; (b) $S=0.75 D$; (c) $S=1.25 D$; (d) $S=1.75 D$; and (e) $S=2.25 D$. Relationships between the dimensionless pedestrian-level mean wind speed and the podium height, street canyon width, and measurement positions when the wind angle is $\theta=0^{\circ}$. 
Table 1. Dimensionless mean wind speed and root-mean-square (RMS) results in the case with $S=0.375 D$.

\begin{tabular}{|c|c|c|c|c|c|c|c|c|}
\hline & Y/D & & $\mathbf{0}$ & 0.5 & 1 & 1.5 & 2 & 2.5 \\
\hline \multirow{8}{*}{$\mathbf{P}_{\mathbf{h}} / \mathbf{D}$} & \multirow{2}{*}{0.5} & Mean & 0.722 & 0.616 & 0.664 & 0.626 & 0.585 & 0.559 \\
\hline & & RMS & 0.427 & 0.245 & 0.163 & 0.206 & 0.298 & 0.292 \\
\hline & \multirow{2}{*}{1} & Mean & 0.767 & 0.691 & 0.666 & 0.573 & 0.502 & 0.470 \\
\hline & & RMS & 0.394 & 0.251 & 0.178 & 0.239 & 0.317 & 0.280 \\
\hline & \multirow{2}{*}{1.5} & Mean & 0.857 & 0.818 & 0.771 & 0.657 & 0.580 & 0.519 \\
\hline & & RMS & 0.406 & 0.332 & 0.275 & 0.364 & 0.455 & 0.373 \\
\hline & \multirow{2}{*}{2} & Mean & 0.898 & 0.868 & 0.823 & 0.711 & 0.627 & 0.544 \\
\hline & & RMS & 0.454 & 0.376 & 0.352 & 0.422 & 0.507 & 0.421 \\
\hline
\end{tabular}

Blocken et al. [3] proposed that three different regimes can be distinguished at the pedestrian level: (1) resistance flow; (2) interaction flow; and (3) isolated flow. Resistance flows occur in narrow passages (e.g., $2 \mathrm{~m}$ in [3]) with high flow resistance in the passage. No flow separation in the passage is observed. Interaction flows occur in wide passages (e.g., $10 \mathrm{~m}$ in [3]), indicating that the two corner streams originating and separating at the passage entrance corners interact and merge together into a single wide passage jet, causing amplification factors higher than those in a single corner stream. Isolated flows (e.g., $30 \mathrm{~m}$ in [3]) mean that there is no interaction of the corner streams in the passage. As revealed from Figure $3 \mathrm{a}$, when $S=0.375$ and $D=7.5 \mathrm{~m}$, the maximum dimensionless mean speed is near the entrance of the street, and the wind speed reduced gradually toward the downstream of the street canyon. This trend is similar to the patterns in the study of Blocken et al. [3] when $W=2 \mathrm{~m}$, which is classified as resistance flow. Although the wind speed measurement results of $S=0.75 D=15 \mathrm{~m}$ and $S=1.25 D=25 \mathrm{~m}$ are classified as interaction flow, the maximum wind speeds appeared near the entrance to the street canyon, and the locations were at approximately $Y=0.5 D$ ( $Y$ is the length of the street canyon), slightly toward the downstream compared to the previous resistance flow. The phenomena were identical to the interaction flow pattern reported in the study [3], as shown in Figure 3b,c. The flow patterns at $S=1.75 D=35 \mathrm{~m}$ and $S=2.25 D=45 \mathrm{~m}$ are classified as isolation flow. As shown in Figure 3d,e, the figure patterns were relatively smooth; due to the larger street canyon width, the wind flows of the center row position were not affected by the buildings on both sides. When comparing the resistance flow and the interaction flow, there was no phenomenon of a sudden increase in the wind speed.

In previous findings, resistance flow existed when the street canyon width was $S=0.375 D=7.5 \mathrm{~m}$; interaction flow existed when the widths of the street canyon were $S=0.75 D$ and $1.25 D=15 \mathrm{~m}$ and $25 \mathrm{~m}$; and isolation flow existed when the widths of the street canyon were $S=1.75 D=35 \mathrm{~m}$ and $2.25 \mathrm{D}=45 \mathrm{~m}$. However, the definitions for the street canyon width are different from those in the previous study [3]. The main reason is that the buildings simulated along the two sides of the street canyon in this study had façades of different sizes (asymmetry), whereas Blocken et al. [3] adopted symmetrical facades. In [3], when the wind flows are blocked from moving toward the street canyon by buildings, two corner vortexes with the same impact ability will converge at the entrance and then enter the street canyon. In this study, when the wind flows enter a street canyon with asymmetrical facades, the corner vortexes formed also have different impact ranges because the two buildings have 
upwind facades with different dimensions. Therefore, the asymmetrical corner vortexes in this study would naturally vary in the wind flow inside the street canyon compared to the symmetrical corner vortexes presented in the literature.

\subsection{Effect of the Podium Height}

When outdoor air flows by a high-rise building, a flow separation occurs at a certain height of the building facade. Part of the airflow crosses upward over the building; some part moves toward both sides; and another large part of the flow moves downward and forms a downwash flow, and a horseshoe vortex is subsequently generated at the bottom of the upwind side, moving toward both sides of the corner. This vortex and another wind flow at the corner combine and generate instantaneous strong wind. This strong corner wind will become stronger with increasing building height.

In this study, the podium width of the main building was $2.5 D$; the width of the attached house was $1 D$. If the street canyon width was under the aforementioned interaction flow, after the approaching wind enters the street canyon, it will be drawn into the street canyon by the corner vortex of the podium. This corner vortex intensity will be affected by the height of the podium (or high-rise building), causing different wind speed changes inside the street canyon. Figure 4 shows the changes in the pedestrian-level wind speed inside the street canyon when the podium heights $\left(P_{\mathrm{h}}=0.5 D, 1 D\right.$, $1.5 D$, and $2 D$ ) and the widths of the street canyons are different; $S=\infty$ indicates that the test conditions have no adjacent low-rise buildings. Our observation shows that as the podium height increases, the magnitude of the overall wind speed inside the street canyon increases accordingly. The effects of the street canyon width on the wind speed are more apparent when $P_{\mathrm{h}} \geq 1 D$.

Figure 4a shows the results for $P_{\mathrm{h}}=0.5 D$, in which the podium height is lower than the height of the attached houses. The dimensionless mean wind speed for the street canyon entrance decreases with increasing street canyon width, whereas the wind speed inside the street canyon downstream was not affected by the street canyon width. The corner vortexes collectively entering the street canyon were relatively weak due to the approaching upwind airflow that mainly crossed over the podium. Two adjacent parallel buildings formed a resistance when the street canyon width was small.

Figure $4 \mathrm{~b}$ shows the results for $P_{\mathrm{h}}=1 D$, in which the podium height is equal to the height of the attached low-rise houses. Compared to Figure $4 \mathrm{a}\left(P_{\mathrm{h}}=0.5 D\right)$, Figure $4 \mathrm{~b}$ shows that there were slight changes in the wind flow characteristic of the street canyon with different widths. The wind speed in the entire street canyon increase, and the downstream wind speed inside the street canyon began to change. When the street canyon width was $S=0.375 D$, the mean wind speed was the highest at the entrance and gradually decreased as it approached the downstream of the street canyon. The mean wind speeds at the street canyon entrance were lower when the widths of the street canyon were $S=0.75 D$ and $1.25 D$; the wind speeds were the highest at the distance of $Y=0.5 D$ away from the entrance and then gradually decreased along the street canyon downstream. 


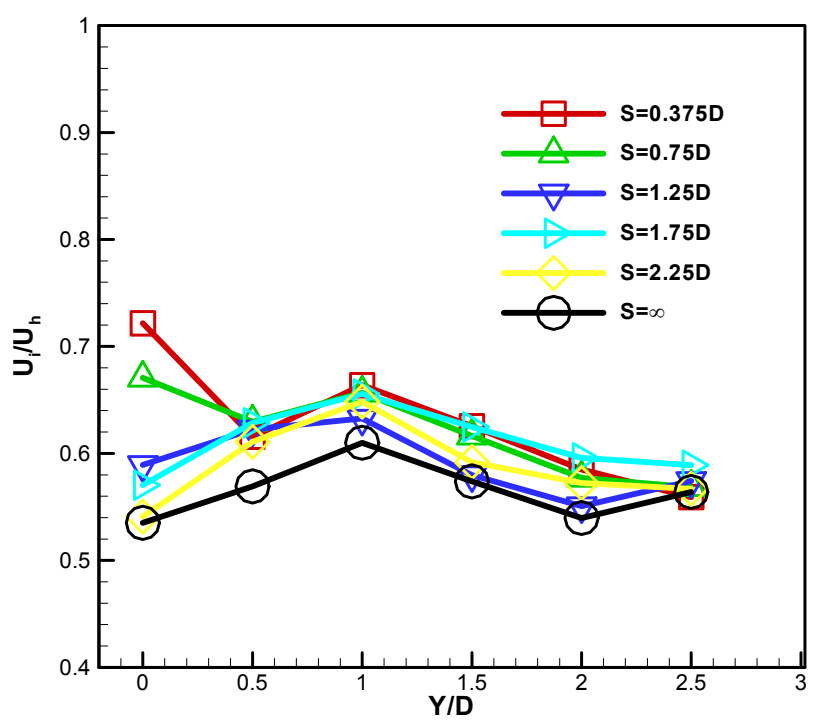

(a)

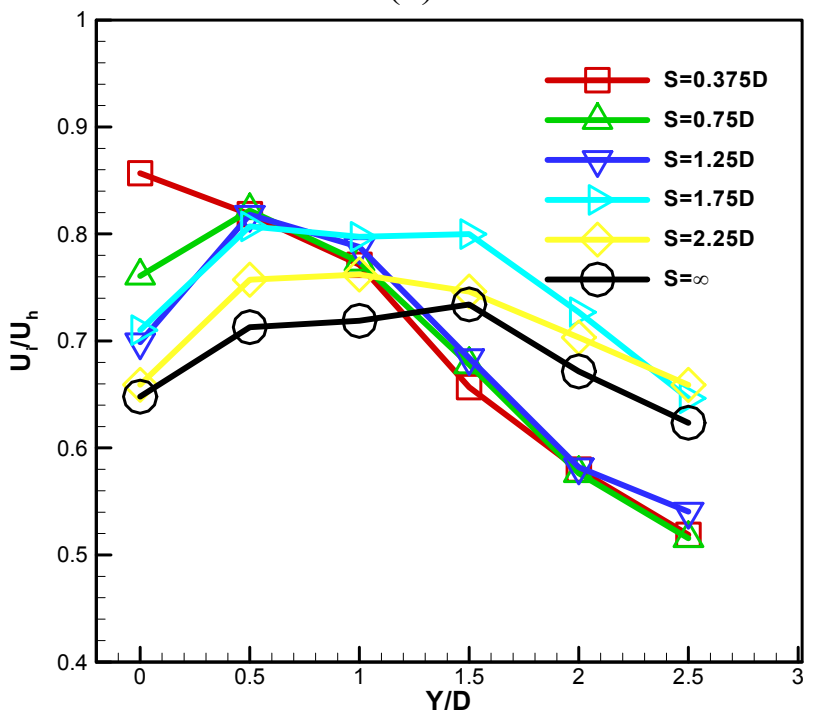

(c)

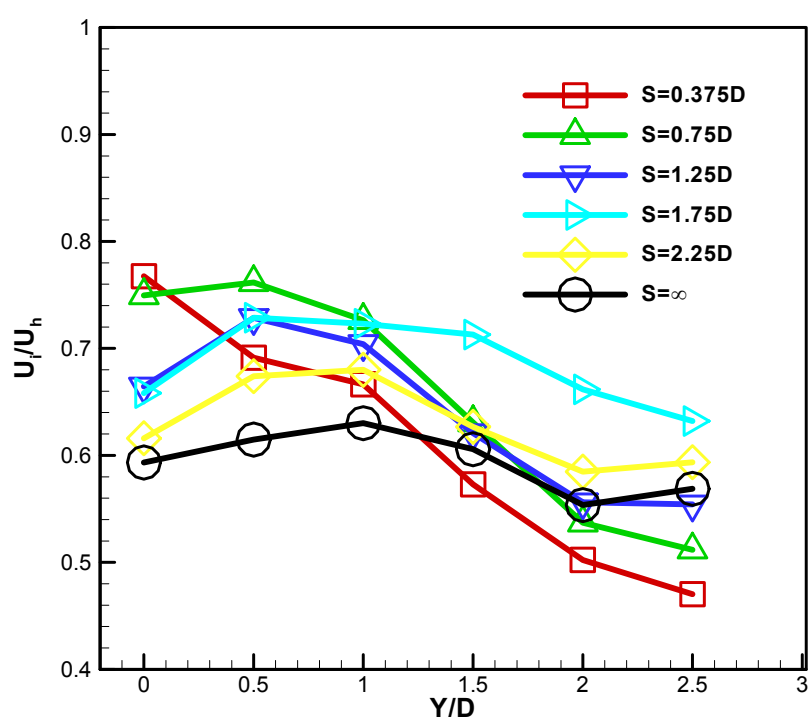

(b)

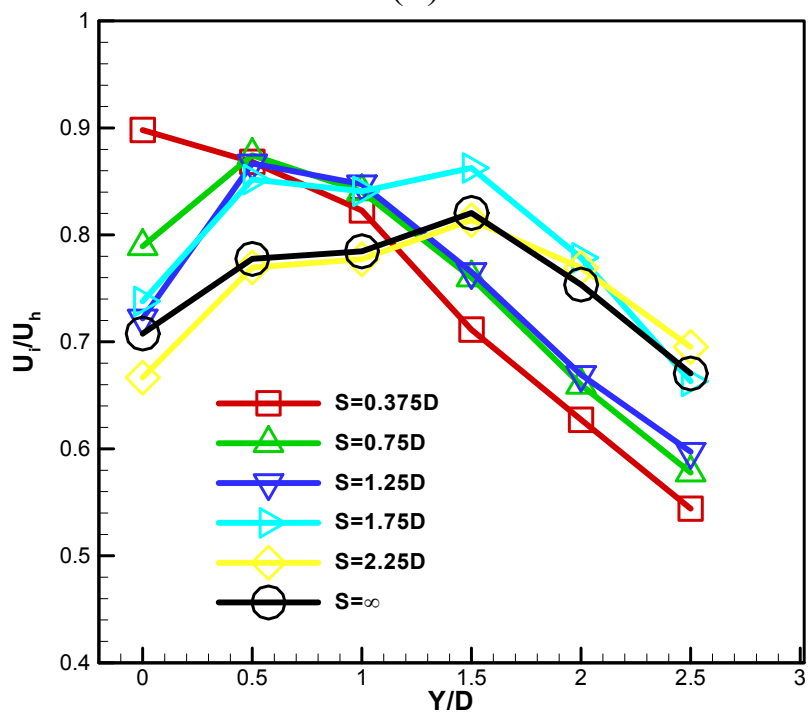

(d)

Figure 4. (a) $P_{\mathrm{h}}=0.5 D$; (b) $P_{\mathrm{h}}=1 D ;$; (c) $P_{\mathrm{h}}=1.5 D$; and (d) $P_{\mathrm{h}}=2 D$. Relationships between the dimensionless mean wind speed and the podium height, street canyon width, and measurement position when the wind direction is $\theta=0^{\circ}$ (continued).

When the widths of the street canyon were $S=1.75 D$ and $2.25 D$, the wind speed characteristics were distinctively different from those in the aforementioned widths. In the midstream of the street canyon, there were segments between $0.5 \mathrm{D}$ and $1.5 \mathrm{D}$ where the wind speeds were higher than those upstream and downstream. When $P_{\mathrm{h}}=2.5 D$ and $P_{\mathrm{h}}=2 D$, the wind speed characteristics inside each type of street canyon width were similar; however, as the podium height in the case of $P_{\mathrm{h}}=2.5 D$ has the highest wind speed, the wind flow generated by the corner vortexes entering the street canyon were relatively high and all of the aforementioned wind speed characteristics were more distinctive, as shown in Figure 4d. When the street canyon widths were $S=2.25 D$ and $S=\infty$, the wind speed plot lines were nearly overlapping; this phenomenon also once again confirms that when the street canyon width was $S=2.25 D=45 \mathrm{~m}$, the flow was completely not affected by the attached low-rise house and was thus isolation flow. 
To comprehensively understand the pedestrian-level wind flow characteristics in the street canyon, this study took the contour plots to represent the wind speed distributions at the upstream, midstream, and downstream areas. As shown in Figures 5-10, the numbers in the plots represent dimensionless mean wind speed, colors closer to blue indicate lower wind speeds, and colors closer to red indicate stronger wind speeds.

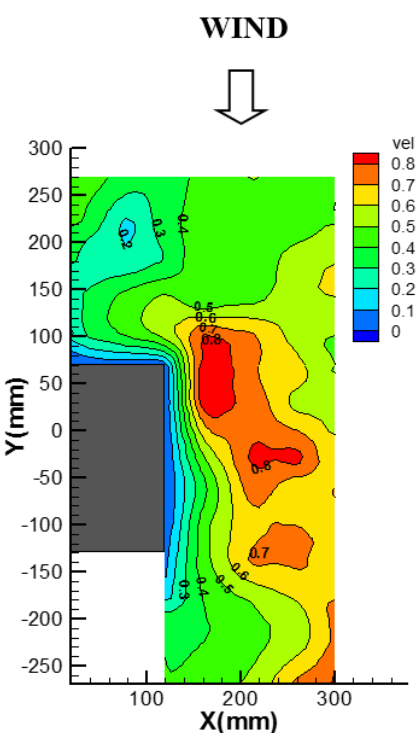

(a)
WIND
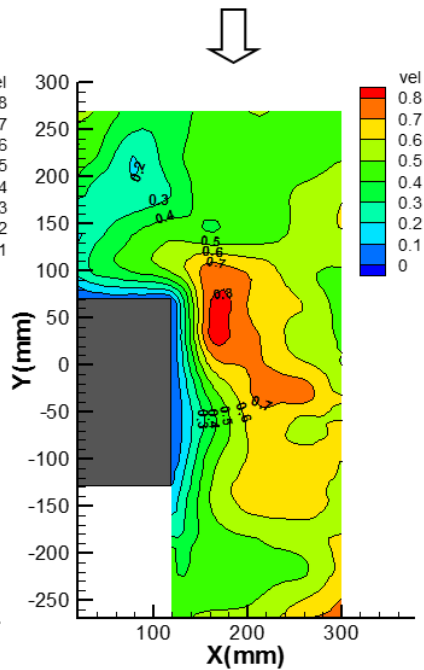

(b)
WIND



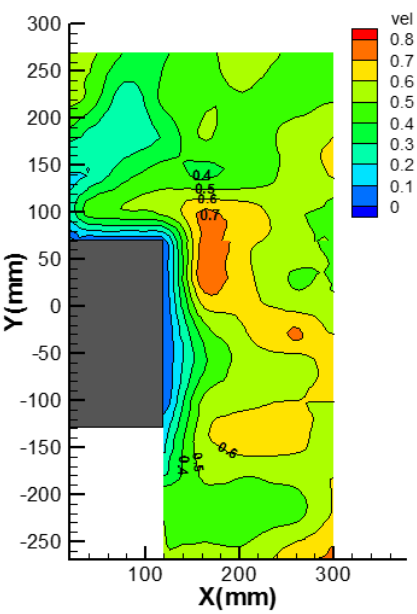

(c)
WIND
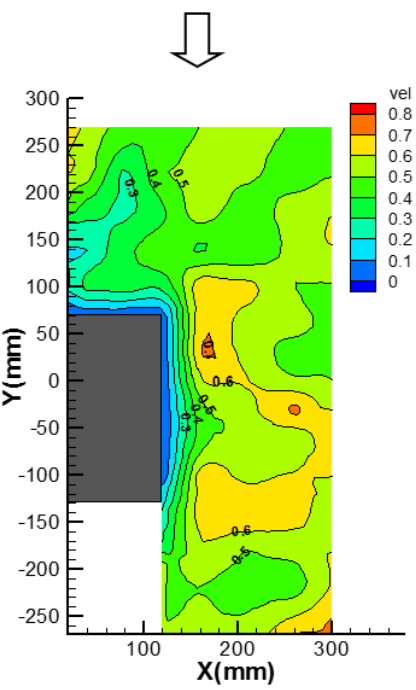

(d)

Figure 5. Dimensionless mean wind speed distributions when there is no street canyon on the side and $\theta=0^{\circ}$ : (a) $P_{\mathrm{h}}=2 D ;\left(\right.$ b) $P_{\mathrm{h}}=1.5 D ;$ (c) $P_{\mathrm{h}}=1 D$; and (d) $P_{\mathrm{h}}=0.5 D$.

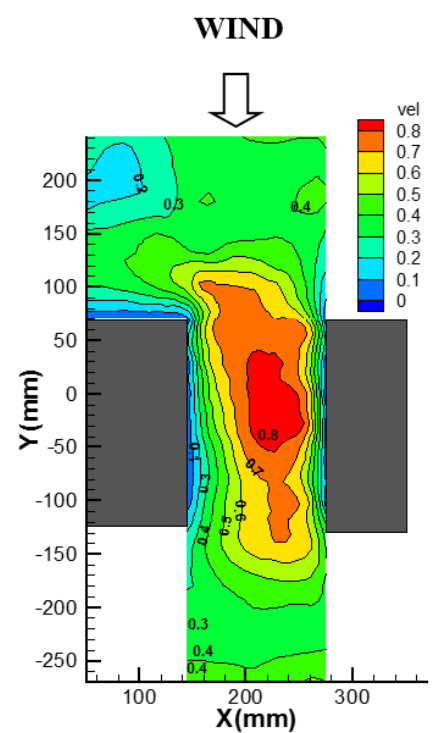

(a)

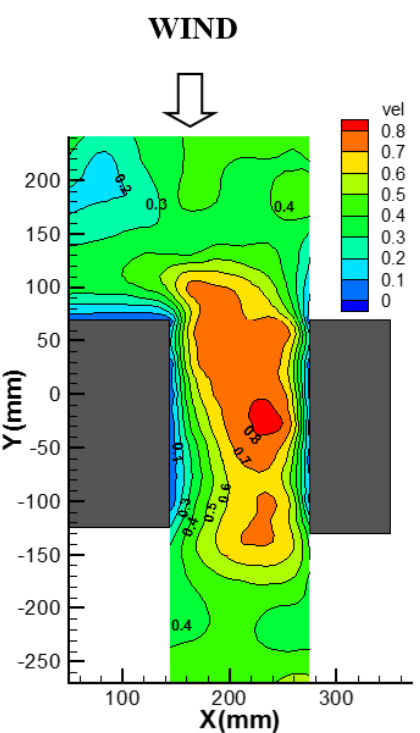

(b)

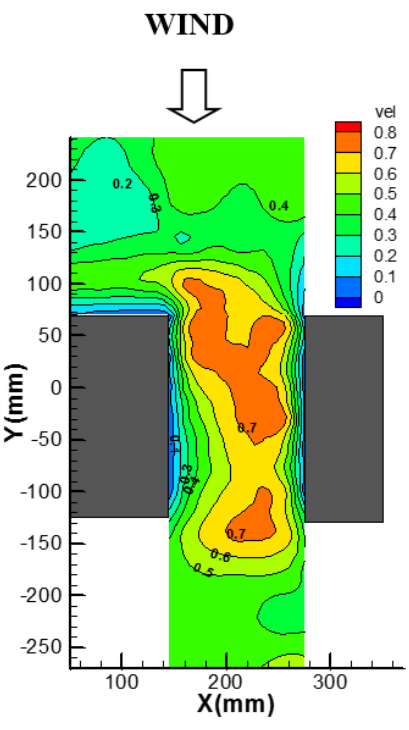

(c)

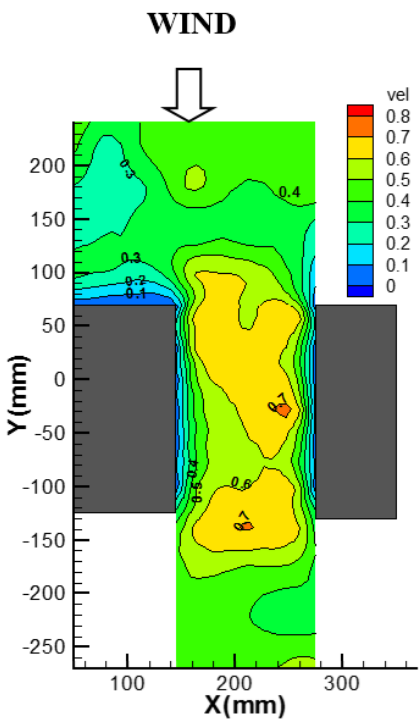

(d)

Figure 6. (a) $P_{\mathrm{h}}=2 D ;$ (b) $P_{\mathrm{h}}=1.5 D ;$ (c) $P_{\mathrm{h}}=1 D$; and (d) $P_{\mathrm{h}}=0.5 D$. Dimensionless mean wind speed distributions when $S=1.75 D$ and $\theta=0^{\circ}$. 
Figure 5 shows the wind speed contours for a standalone high-rise building with a podium at an angle of $0^{\circ}$. Figure 6 shows the pedestrian-level wind flow characteristics in the street canyon when $S=1.75 D$. The podium height in Figure 6a was the highest, so the zone of emerging high wind speed in the street canyon was the largest; the wind speed also decreases with a lower podium height (Figure $6 \mathrm{~b}-\mathrm{d}$ ). The high wind speed positions in Figures 5 and 6 were slightly different. In Figure 5, the main high wind speed focus area was at the corner, whereas in Figure 6, it appeared inside the street canyon. The main reason for this difference is the narrowness of the street canyon. The wind speed inside the smaller passage was suddenly accelerated because of the Venturi effect, causing the so-called channeling effect.

\subsection{Effect of the Approaching Wind Direction}

Figure 7 shows the wind speed distributions for the street canyon of $S=1.75 D$ and $\theta=22.5^{\circ}$. Because the downwash flow intensity on the upwind side became smaller, the approaching flow entering the street corner was not parallel to the street canyon, so the wind speed at the street canyon upstream zone was significantly weaker than that when $\theta=0^{\circ}$. Moreover, the side of the podium formed a barrier to the approaching wind. Part of the wind flow moved along the side of podium toward the street canyon downstream, whereas the other parts formed the downwash flow, leading to a higher wind speed in the street canyon downstream. The intensity and range of this wind flow were controlled by the podium height; a higher podium resulted in greater downwash flow.

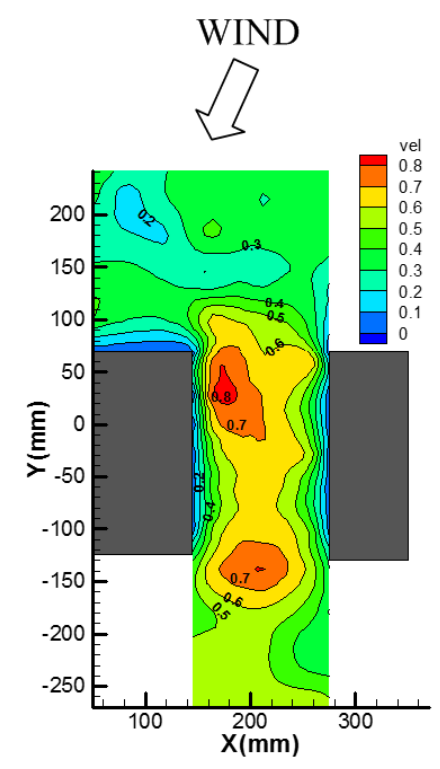

(a)

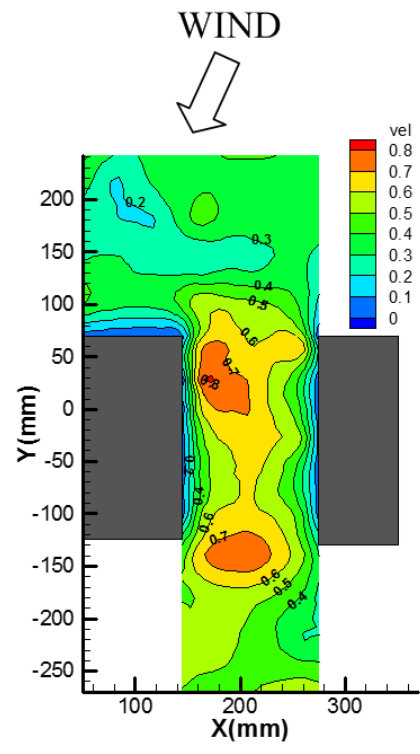

(b)

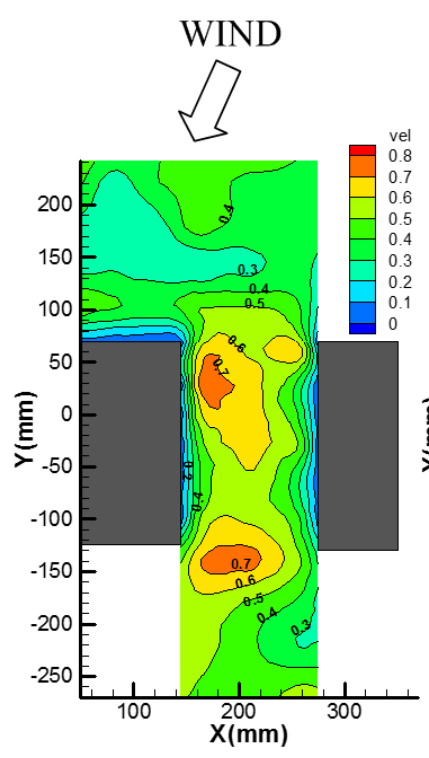

(c)

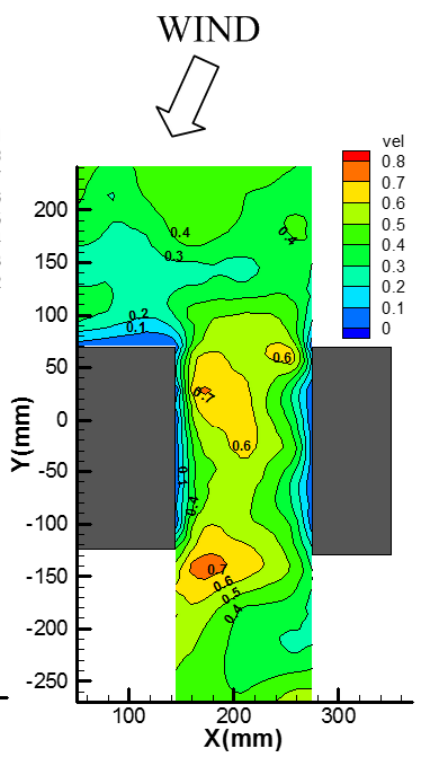

(d)

Figure 7. Dimensionless wind speed distributions when $S=1.75 D$ and $\theta=22.5^{\circ}$ : (a) $P_{\mathrm{h}}=2 D$; (b) $P_{\mathrm{h}}=1.5 D ;$; (c) $P_{\mathrm{h}}=1 D$; and (d) $P_{\mathrm{h}}=0.5 D$.

Figure $7 \mathrm{a}, \mathrm{b}$ shows the scenarios when the podium height was greater than the height of the adjacent low-rise attached houses; the wind flow formed scenarios similar to the vertical horseshoe vortex in the street canyon downstream. In contrast, Figure $7 \mathrm{c}, \mathrm{d}$ shows the scenarios when the podium height was lower than or equal to those of the adjacent attached houses. For the street canyon downstream, 
there was no downwash flow; the wind flow only took a $22.5^{\circ}$ angle to cut through the adjacent attached houses, which is why the range of the high wind speed zone was smaller.

The aforementioned wind flow characteristics in street canyons downstream were more significant when the wind direction was at $\theta=45^{\circ}$. The blockage formed by the side of the podium on the approaching wind flow was larger; the downwash flow generated was also stronger. When the podium was higher than the adjacent low-rise attached houses, a stronger wind speed field was generated downstream of the street canyon. As shown in Figure 8a,b, the mean wind speeds were significantly higher than those in Figure 7a,b and the affecting ranges were greater. In Figure 8b,c, the downwash flow effects generated by the podium obstruction were smaller because the podium heights were higher than or equal to the height of the adjacent attached houses, respectively.

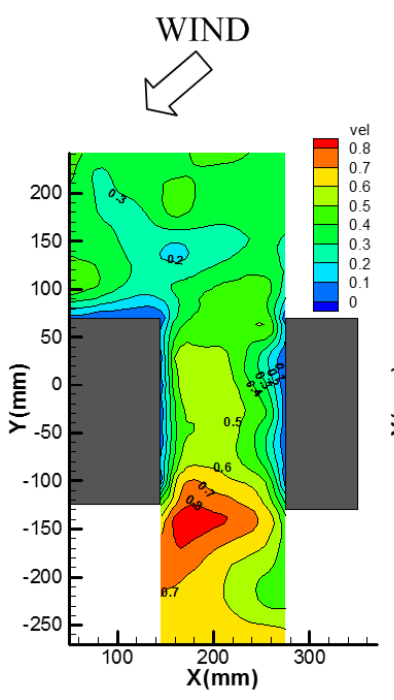

(a)

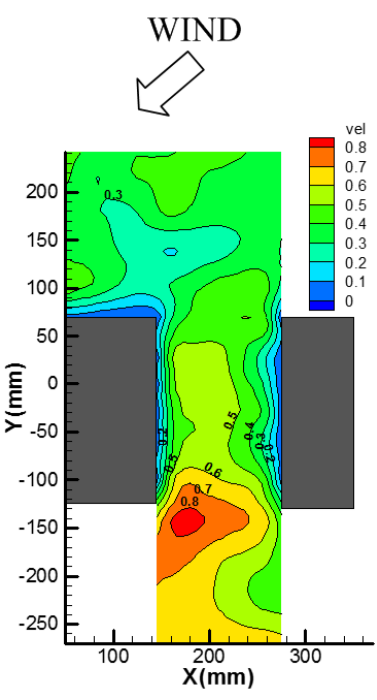

(b)

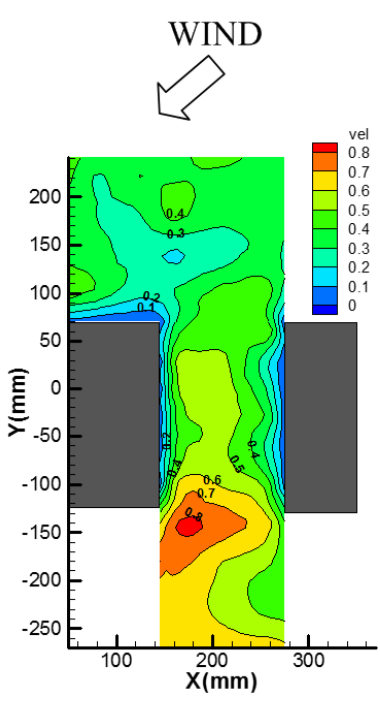

(c)

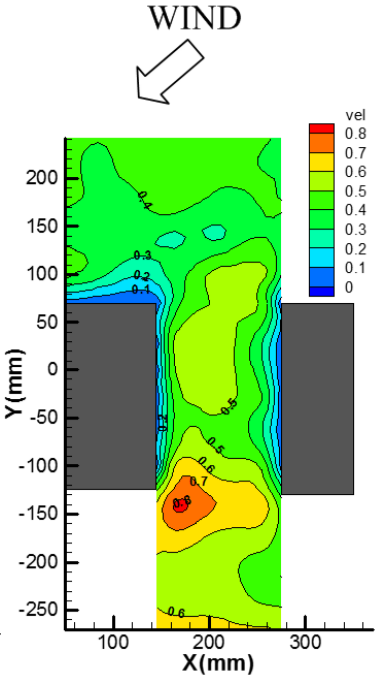

(d)

Figure 8. Dimensionless mean wind speed distributions when $S=1.75 D$ and $\theta=45^{\circ}$ : (a) $P_{\mathrm{h}}=2 D ;\left(\right.$ b) $P_{\mathrm{h}}=1.5 D ;$ (c) $P_{\mathrm{h}}=1 D$; and (d) $P_{\mathrm{h}}=0.5 D$.

Figure 9 shows the interaction of approaching wind on the upwind side of the building at $-22.5^{\circ}$. The wind flow obstructed by the podium flowed into the street canyon along the podium, but the wind was guided toward the outside when the wind flow encountered the adjacent attached low-rise houses. The wind flow entering the street canyon is affected by the podium, but it is weaker than in the scenario of $\theta=0^{\circ}$. Most of the wind flow in the street canyon upstream moved along the front end of the podium entering into the street canyon, and the corner vortexes were also affected by the podium height. As shown in Figure 9, as the podium height decreased, the wind speeds of the street canyon upstream also gradually decreased. However, the wind flow in the street canyon downstream had the opposite trend. In terms of a wind direction of $-22.5^{\circ}$, the podium formed a blockage on the wind flow, forcing the wind to channel through the passage following a long side of the attached houses toward the downstream, as shown in Figure 9a,b. However, when the podium height was gradually decreased, the wind could flow across the podium at the street canyon exit and the flow collection inside the street canyon was as shown in Figure 9c. When the podium height was less than the adjacent attached houses, the wind flow over the podium was blocked and deflected by the adjacent attached houses, creating high wind and turbulence, as shown in Figure 9d. 


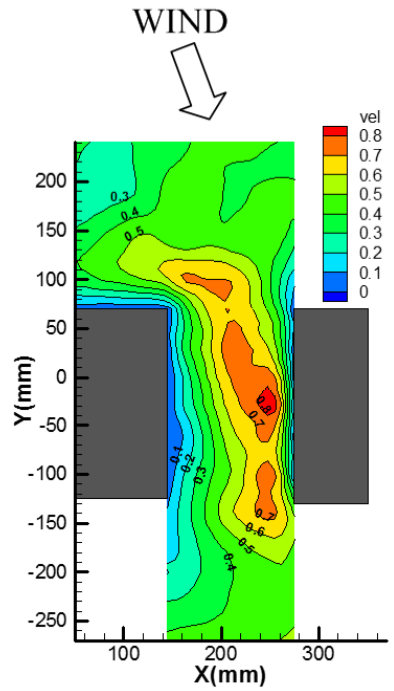

(a)

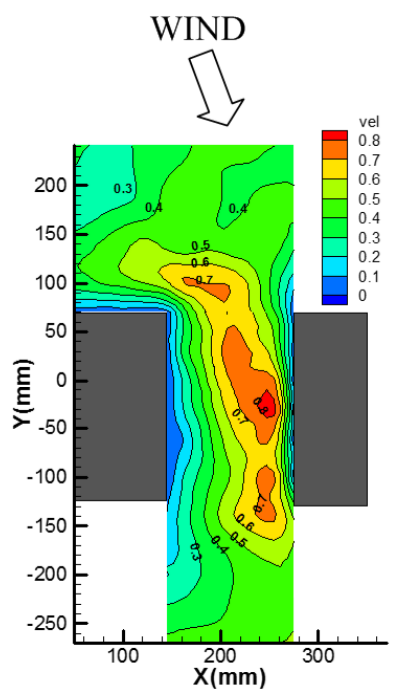

(b)

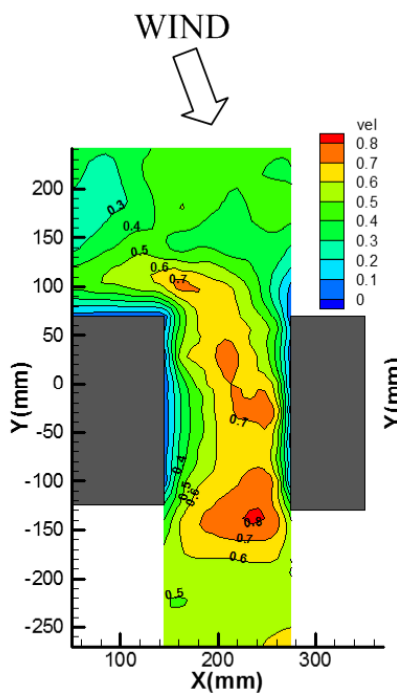

(c)

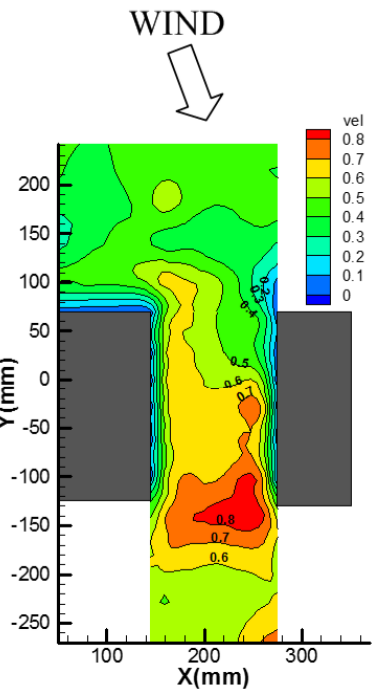

(d)

Figure 9. Dimensionless mean wind speed distributions when $S=1.75 D$ and $\theta=-22.5^{\circ}$ :

(a) $P_{\mathrm{h}}=2 D ;\left(\right.$ b) $P_{\mathrm{h}}=1.5 D ;\left(\right.$ c) $P_{\mathrm{h}}=1 D$; and (d) $P_{\mathrm{h}}=0.5 D$.

When the wind direction was changed to $-45^{\circ}$, as shown as Figure 10, the downwash flows of the upwind side were stronger than those with the wind direction of $-22.5^{\circ}$. Therefore, a higher wind speed area appeared both upstream and downstream. Moreover, comparing Figures 9 and 10, when the wind direction of $-45^{\circ}$ was larger, the high wind speed in the street canyon moved further toward the adjacent attached low-rise houses.

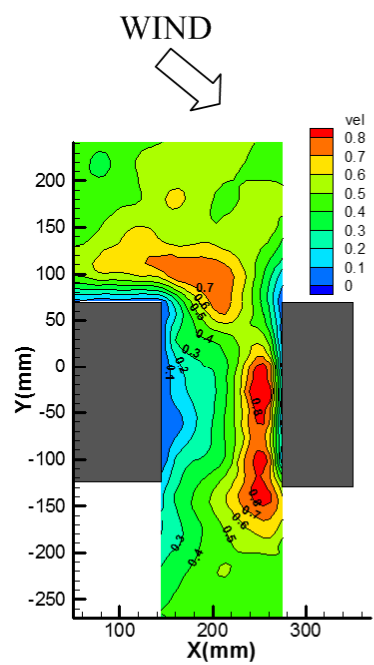

(a)

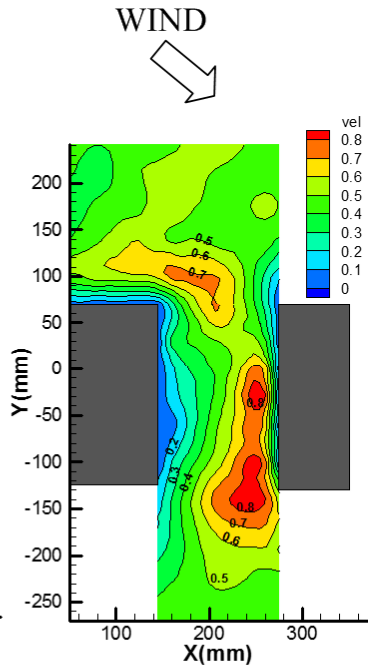

(b)

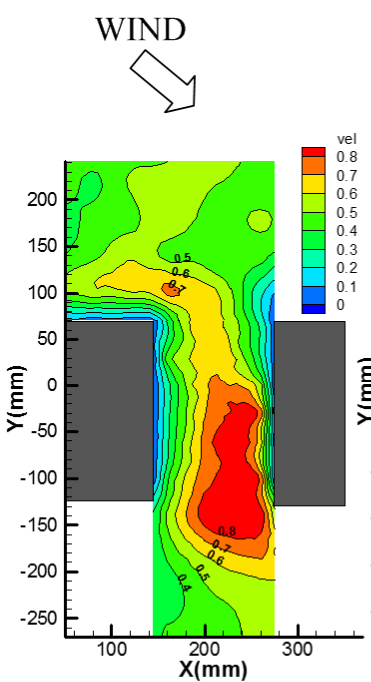

(c)

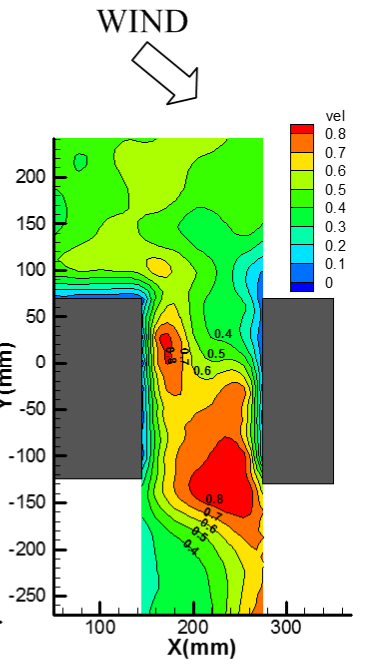

(d)

Figure 10. (a) $P_{\mathrm{h}}=2 D ;\left(\right.$ b) $P_{\mathrm{h}}=1.5 D ;$ (c) $P_{\mathrm{h}}=1 D$; and (d) $P_{\mathrm{h}}=0.5 D$. Dimensionless mean wind speed distributions when $S=1.75 D$ and $\theta=-45^{\circ}$.

\section{Conclusions}

Taking the street canyon formed by a high-rise building with a podium and the adjacent attached low-rise houses as the main controllers, this study explored the pedestrian-level wind flow characteristics 
inside the street canyon. The variables included the street canyon width, approaching wind direction, and podium height. The following conclusions were obtained:

(1) This study took street canyon widths of $S=0.375 D, 0.75 D, 1.25 D, 1.75 D$, and $2.25 D$ to conduct the testing. After comparing the dimensionless mean wind speed at the street canyon center with the literature, it was concluded that a street canyon with $S=0.375 D$ exhibited resistance flow; those with $S=0.75 D$ and $1.25 D$ were considered interaction flow; and those with $S=1.75 D$ and $2.25 D$ were considered isolation flow.

(2) Podium height controlled the wind flow characteristics of the street canyon. Regardless of the width of the street canyon, all of the mean wind velocities were higher than the wind velocities before entering the street canyon, and there were significant channeling effects. The wind inside the street canyon was greatly affected by the podium height. The wind speed inside the street canyon was greater for higher podiums.

(3) When the wind direction was $0^{\circ}$, the maximum wind speeds mainly appeared at the front end of the street canyon. As the wind angle gradually changed and the podium height became greater than that of the attached houses, the maximum wind speed still appeared at the front end of street canyon, but the maximum wind speed in the street canyon appeared downstream when the podium height was less than the height of the attached houses.

(4) The test model in this study was an asymmetrical configuration. Hence, although the wind direction angles of $\pm 22.5^{\circ}$ and $\pm 45^{\circ}$ were taken as the angle of symmetry, the attained wind characteristics in the street canyon were vastly different. When the wind angle was $22.5^{\circ}$, there were high-wind-speed zones both upstream and downstream of the street canyon; when it was at $45^{\circ}$, the high-wind-speed zone appeared in the downstream of the street canyon. When the wind angle was at $-22.5^{\circ}$ or $-45^{\circ}$, the wind flow was blocked by the podium. Higher podiums resulted in a smaller high-wind-speed area; conversely, lower podiums resulted in larger high-wind-speed areas.

\section{Acknowledgments}

Support from the Architecture and Building Research Institute, Ministry of the Interior, Taiwan through Grant No. PG10205-0122 in this study is gratefully acknowledged.

\section{Author Contributions}

Chien-Yuan Kuo and Ming-Chin Ho conceived of and designed the experiments; Chien-Yuan Kuo and Chun-Ta Tzeng performed the experiments; Chien-Yuan Kuo and Chi-Ming Lai analyzed the data; and Chien-Yuan Kuo and Chi-Ming Lai wrote the paper.

\section{Conflicts of Interest}

The authors declare no conflicts of interest. 


\section{References}

1. To, A.P.; Lam, K.M. Evaluation of pedestrian-level wind environment around a row of tall buildings using a quartile-level wind speed descriptor. J. Wind Eng. Ind. Aerodyn. 1995, 54, 527-541.

2. Stathopoulos, T.; Wu, H.; Bédard, C. Wind environment around buildings: A knowledge-based approach. J. Wind Eng. Ind. Aerodyn. 1992, 44, 2377-2388.

3. Blocken, B.; Carmeliet, J.; Stathopoulos, T. CFD evaluation of wind speed conditions in passages between parallel buildings-effect of wall function roughness modifications for the atmospheric boundary layer flow. J. Wind Eng. Ind. Aerodyn. 2007, 95, 941-962.

4. Blocken, B.; Stathopoulos, T.; Carmeliet, J. Wind environmental conditions in passages between two long narrow perpendicular buildings. J. Aerosp. Eng. 2008, 21, 280-287.

5. Tsang, C.W.; Kwok, K.C.S.; Hitchcock, P.A. Wind tunnel study of pedestrian level wind environment around tall buildings: Effects of building dimensions, separation and podium. Build. Environ. 2012, 49, 167-181.

6. Gu, Z.L.; Zhang, Y.W.; Cheng, Y.; Lee, S.C. Effect of uneven building layout on air flow and pollutant dispersion in non-uniform street canyon. Build. Environ. 2011, 46, 2657-2665.

7. Hang, J.; Li, Y.; Sandberg, M.; Buccolieri, R.; Di Sabatino, S. The influence of building height variability on pollutant dispersion and pedestrian ventilation in idealized high-rise urban areas. Build. Environ. 2012, 56, 346-360.

8. Moon, K.; Hwang, J.M.; Kim, B.G.; Lee, C.; Choi, J.I. Large-eddy simulation of turbulent flow and dispersion over a complex urban street canyon. Environ. Fluid Mech. 2014, 14, 1381-1403.

9. Ramponi, R.; Blocken, B.C.L.B.; Janssen, W.D. CFD simulation of outdoor ventilation of generic urban configurations with different urban densities and equal and unequal street widths. Build. Environ. 2015, 92, 152-166.

10. Wind Resistance Design Specifications and Commentary of Buildings; Ministry of the Interior: Taipei City, Taiwan, 2014. (In Chinese)

11. Irwin, H.P.A.H. A simple omnidirectional sensor for wind tunnel studies of pedestrian-level winds. J. Wind Eng. Ind. Aerodyn. 1981, 7, 219-239.

12. Wu, H.; Stathopoulos, T. Further experiments on Irwin's surface wind sensor. J. Wind Eng. Ind. Aerodyn. 1994, 53, 441-452.

13. Soligo, M.J.; Irwin, P.A.; Willaims, C.J.; Schuyler, G.D. A comprehensive assessment of pedestrian comfort including thermal effects. J. Wind Eng. Ind. Aerodyn. 1998, 77, 753-766.

14. Outdoor Human Comfort and Its Assessment; Technical Report for the Aerospace Division of American Society of Civil Engineers (ASCE): Reston, VA, USA, 2004.

(C) 2015 by the authors; licensee MDPI, Basel, Switzerland. This article is an open access article distributed under the terms and conditions of the Creative Commons Attribution license (http://creativecommons.org/licenses/by/4.0/). 